\title{
Stressed DCs can't handle T cells
}

\section{$c 6$ \\ $\mathrm{XBP} 1$ \\ activation ... \\ disrupts lipid \\ homeostasis \\ in tDCs}

Tumour cells adapt to the adverse conditions in which they must survive - such as hypoxia, nutrient starvation and oxidative stress - by triggering an endoplasmic reticulum (ER) stress response, which directly supports tumorigenesis. Glimcher and colleagues now show that the ER stress response in tumour-associated dendritic cells (tDCs) indirectly promotes tumorigenesis by inhibiting the activation of an antitumour $\mathrm{T}$ cell response.

Activation of the ER stress sensor IRE 1 a leads to splicing of XBP1 mRNA to generate functional XBP1 transcription factor. In mice with

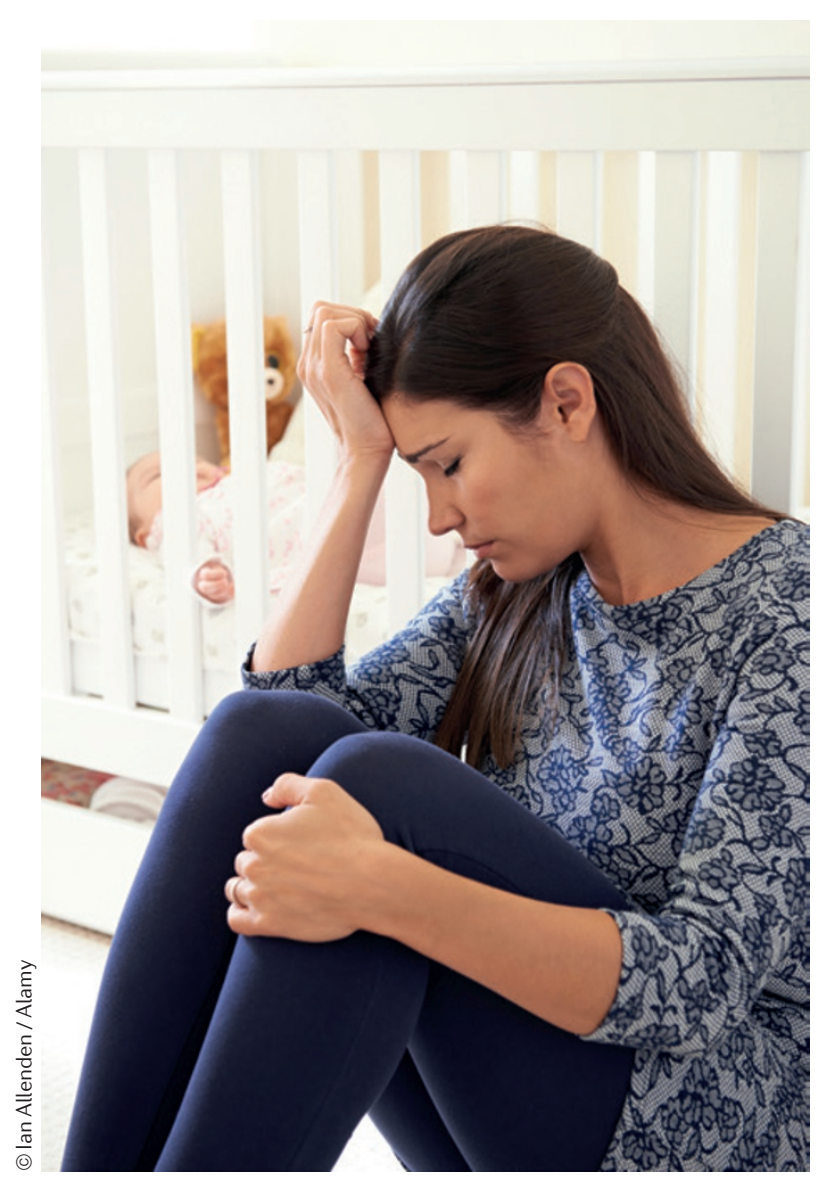

aggressive primary and metastatic ovarian cancers, $X b p 1$ splicing was markedly enhanced in tDCs compared with splenic DCs (sDCs), and this was associated with increased nuclear XBP1 protein and upregulation of expression of XBP1 target genes and general ER stress response markers. Similar observations were made for tDCs isolated from human ovarian cancer samples. When XBP1 was conditionally deleted in DCs in three preclinical models of primary and metastatic ovarian cancer, tumour growth and spread were significantly impaired and median survival was increased. Thus, XBP1 activation in $\mathrm{tDCs}$ accelerates tumour progression, but what are the cause and effect of ER stress in tDCs?

Ovarian cancer-associated DCs had significantly higher levels of intracellular lipids and of reactive oxygen species (ROS) than control sDCs. Lipid oxidation by ROS is known to generate 4-hydroxytrans-2-nonenal (4-HNE), which induces ER stress by forming protein adducts. Increased levels of 4 -HNE were observed in $\mathrm{tDCs}$ compared with sDCs, and sDCs exposed to cell-free peritoneal fluid from ovarian cancer-bearing hosts rapidly generated intracellular 4-HNE protein adducts. Confirming that lipid oxidation is the cause of ER stress in tDCs, treatment of freshly isolated $\mathrm{tDCs}$ with vitamin $\mathrm{E}$ (an ROS-scavenging agent) or with hydralazine (which sequesters lipid peroxidation byproducts) inhibited $X b p 1$ splicing and the induction of ER stress response markers.

Transcriptional profiling showed that XBP1-deficient tDCs have downregulated expression of genes involved in triglyceride biosynthesis, and these genes could be rapidly induced in naive sDCs exposed to 4-HNE. The intracellular lipid content of tDCs was decreased by XBP1 deficiency or pharmacological inhibition, or by treatment with vitamin $\mathrm{E}$ or hydralazine, which indicates that XBP1 activation in response to lipid oxidation disrupts lipid homeostasis in tDCs.

Finally, the authors showed that intracellular lipid accumulation downstream of XBP1 activation decreases the antigen-presenting capacity of tDCs through decreased surface expression of peptide-MHC complexes and hence reduces their capacity to induce $T$ cell proliferation. tDCs lacking XBP1 or pre-treated with vitamin $\mathrm{E}$ had improved antigen-presenting capacity. Furthermore, T cells obtained from tumour-bearing mice with a DC-specific XBP1 deficiency had increased capacity to restrain tumour growth in adoptively transferred hosts compared with T cells from tumour-bearing wild-type mice.

Together the data indicate that targeting the ER stress response in cancer-bearing hosts could induce indirect (in tDCs) as well as direct (in tumour cells) antitumour mechanisms. Indeed, Xbp1-specific RNA-mediated interference using tDC-targeted nanoparticles in mice with metastatic ovarian cancer decreased triglyceride levels in tDCs, which increased $\mathrm{T}$ cell infiltration of tumours and $\mathrm{T}$ cell activation, and decreased the number of metastatic cancer cells in the peritoneal cavity. Kirsty Minton

ORIGINAL RESEARCH PAPER Cubillos-Ruiz, J. R. et al. ER stress sensor XBP1 controls anti-tumor immunity by disrupting dendritic cell homeostasis. Cell 161, 1527-1538(2015) 